\title{
Explaining the Flavor Anomalies with a Vector Leptoquark (Moriond 2019 update)
}

\section{Andreas Crivellin*}

Paul Scherrer Institut, CH-5232 Villigen PSI, Switzerland

Physik-Institut, Universität Zürich, Winterthurerstrasse 190, CH-8057 Zürich, Switzerland

E-mail: andreas.crivellinecern.ch

\section{Francesco Saturnino ${ }^{\dagger \ddagger}$}

Albert Einstein Center for Fundamental Physics, Institute for Theoretical Physics, University of Bern, CH-3012 Bern, Switzerland

E-mail: saturninoeitp.unibe.ch

\begin{abstract}
Several experiments revealed intriguing hints for lepton flavor universality (LFU) violating new physics (NP) in semi-leptonic $B$ meson decays, mainly in $b \rightarrow c \tau v$ and $b \rightarrow s \ell^{+} \ell^{-}$transitions at the $3-5 \sigma$ level. Leptoquarks (LQ) are prime candidates to address these anomalies as they contribute to semi-leptonic decays already at tree level while effects in other flavor observables, agreeing with the standard model (SM), are loop suppressed.

In these proceedings we review the vector leptoquark $S U(2)_{L}$ singlet, contained in the famous Pati-Salam model, which is able to address both $b \rightarrow c \tau \nu$ and $b \rightarrow s \mu^{+} \mu^{-}$data simultaneously. Due to the large couplings to tau leptons needed to account for the $b \rightarrow c \tau v$ data, sizable loop effects arise which we include in our phenomenological analysis. Updating our result of Ref. [1] with the recent measurements of LHCb [2] and BELLE [3,4] we find an even better fit to data than before.
\end{abstract}

XXVII International Workshop on Deep-Inelastic Scattering and Related Subjects - DIS2019 8-12 April, 2019, Torino, Italy

\footnotetext{
*The work of A.C. is supported by a Professorship Grant (PP00P2_176884) of the Swiss National Science Foundation.

${ }^{\dagger}$ Speaker.

$\ddagger$ I thank the organizers of the DIS2019 in Turin for giving me the opportunity to present my work, which is supported by the Swiss National Foundation under grant 200020_175449/1. We are very grateful to Joaquim Matias and Bernat Capdevilla for providing us with the fit necessary for the $b \rightarrow s \ell^{+} \ell^{-}$region in Fig. 2. These proceedings match the ones of Andreas Crivellin for Moriond QCD \& High Energy Interactions (23-30 March 2019, La Thuile, Italy) and are submitted as a common arxiv version.
} 


\section{Introduction}

While so far the LHC has not detected any particles beyond the ones present in the Standard Model (SM), intriguing hints for LFU violation in semi-leptonic $B$-meson decays were accumulated in several (classes of) observables:

$b \rightarrow s \ell^{+} \ell^{-}$

In these flavor changing neutral current transitions, measurements of the ratios

$$
R\left(K^{(*)}\right)=\frac{\operatorname{Br}\left[B \rightarrow K \mu^{+} \mu^{-}\right]}{\operatorname{Br}\left[B \rightarrow K e^{+} e^{-}\right]}
$$

show sizable deviations form their respective SM prediction. While the newest measurement of $R(K)$ by the LHCb collaboration [2] shows a deviation of $2.5 \sigma$ from the SM, the Belle result for $R\left(K^{(*)}\right)$ is consistent with the SM [3]. However, due to the larger errors, this result also agrees with previous LHCb measurements of $R\left(K^{(*)}\right)$ which deviate from the SM [5] in the same direction as $R(K)$. Taking into account all other $b \rightarrow s \mu^{+} \mu^{-}$observables (like the lepton flavor universal observable $P_{5}^{\prime}$ [6]), the global fit prefers various NP scenarios above the $5 \sigma$ level [7] compared to the SM, also when the newest measurements are taken into account [8-11].

In order to resolve the discrepancy in the neutral current transitions, an effect of $\mathscr{O}(10 \%)$ is required at the amplitude level. Since this flavor changing neutral current (FCNC) is suppressed in the SM as it is only induced at one loop level, a small NP contribution is already sufficient. In a global fit one finds a preference for scenarios like $C_{9}^{\mu \mu}=-C_{10}^{\mu \mu}$ (i.e. a left-handed current coupling to muons only) [8]. Such an effect is naturally obtained at tree-level with the vector LQ $S U(2)$ singlet [1,12-32]. However, a $C_{9}^{\mu \mu}=-C_{10}^{\mu \mu}$ effect complemented by a flavor universal effect in $C_{9}$ gives an even better fit to data $[8,33]$. As we will see, this is exactly the pattern that arises in our model.

$b \rightarrow c \tau v$

There are also indications for LFU violation in charged current transitions, namely in the ratios

$$
R\left(D^{(*)}\right)=\frac{\operatorname{Br}\left[B \rightarrow D^{(*)} \tau \nu\right]}{\operatorname{Br}\left[B \rightarrow D^{(*)} \ell v\right]}
$$

where $\ell=\{e, \mu\}$. While the newest measurements from Belle [4] agree with the SM prediction, including previous measurements by BaBar, Belle and LHCb still yield a deviation of $3.1 \sigma$ [34] from the SM prediction. Furthermore there is also a measurement of the ratio $R(J / \Psi)=\frac{\operatorname{Br}\left[B_{c} \rightarrow J / \Psi \tau v\right]}{\operatorname{Br}\left[B_{c} \rightarrow J / \Psi \mu v\right]}$ exceeding its SM prediction [35].

Also here a NP effect of $\mathscr{O}(10 \%)$ is needed at the amplitude level. However, since $b \rightarrow c \tau v$ transitions are mediated at tree level by the exchange of a $W$ boson in the SM, the NP effect needs to be large. This means that NP should contribute at tree level with sizable couplings and at a not too high NP scale. Here, the best single particle solution is the vector LQ $S U(2)$ singlet [1,12-32] since it does not give a tree-level effect in $b \rightarrow s v v$ processes and provides a common rescaling of $R(D)$ and $R\left(D^{*}\right)$ with respect to the SM prediction. 

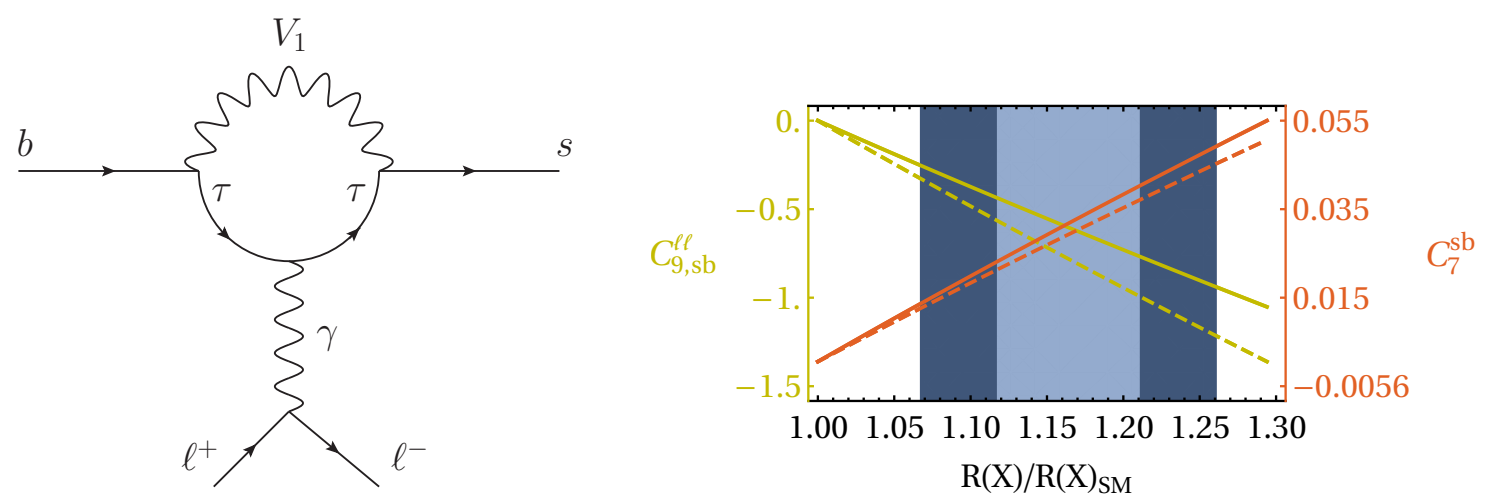

Figure 1: Left: Feynman diagram depicting the loop effects induced by the $b c \tau v$ operator from $S U(2)$ invariance. Right: $C_{9, s b}^{\ell \ell}$ and $C_{7}^{s b}\left(\mu_{b}\right)$, generated by these loop effects, as functions of $R\left(D^{(*)}\right) / R\left(D^{(*)}\right)_{\mathrm{SM}}$. The solid (dashed) lines correspond to $M=1 \mathrm{TeV}(5 \mathrm{TeV})$ while the (dark) blue region is preferred by $b \rightarrow c \tau \nu$ data at the $1 \sigma(2 \sigma)$ level, taking into account the most recent measurements. From the global fit, taking into account only lepton flavor conserving observables, we have $-1.29<C_{9, s b}^{\ell \ell}<-0.87$ [38] and $-0.01<C_{7}^{s b}\left(\mu_{b}\right)<0.05$ [7] at the $1 \sigma$ level. Assuming an explanation of $b \rightarrow c \tau \nu$, our model predicts the right size and sign of the effect in $C_{9, s b}^{\ell \ell}$ and $C_{7}^{s b}\left(\mu_{b}\right)$ needed to explain $b \rightarrow s \ell^{+} \ell^{-}$data.

\section{The Pati Salam vector leptoquark as combined solution to the anomalies}

The vector Leptoquark $S U(2)_{L}$ singlet with hypercharge $-4 / 3$, arising in the famous PatiSalam model [36], is a prime candidate to explain both the anomalies in charged current and neutral current $B$ decays simultaneously [12-14, 17-20]. It gives a $C_{9}=-C_{10}$ effect in $b \rightarrow s \ell^{+} \ell^{-}$at tree level and at the same time a sizable effect in $b \rightarrow c \tau v$ without violating bounds from $b \rightarrow s v v$ and/or direct searches and does not lead to proton decay. Note that this LQ by itself is not UV complete, however several UV complete models for this LQ have been proposed [15, 16, 21-29, 37].

For the purpose of our phenomenological analysis, let us consider a model where we simply extend the SM by this LQ. Its interaction with the SM particles is given by the Lagrangian

$$
\mathscr{L}_{V_{1}}=\kappa_{f i}^{L} \overline{Q_{f}} \gamma_{\mu} L_{i} V_{\mu}^{1^{\dagger}}+\text { h.c. }
$$

where $Q(L)$ is the quark (lepton) $S U(2)_{L}$ doublet, $\kappa_{f i}^{L}$ represents the couplings of the LQ to the left handed quarks (leptons) and $f$ and $i$ are flavor indices. Note that in principle couplings to right-handed SM particles are also allowed, they are however not relevant for this discussion. After electro-weak symmetry breaking, we work in the down basis, meaning that no CKM matrix elements appear in FCNC processes.

We start by taking $\kappa_{23}^{L}$ and $\kappa_{33}^{L}$ as the only non-zero couplings, as they are necessary to explain $b \rightarrow c \tau v$ data. Here, strong effects in $b \rightarrow s \tau^{+} \tau^{-}$transitions [39] are generated which at the 1-loop level affect $b \rightarrow s \ell^{+} \ell^{-}$via the Wilson coefficients $C_{9, s b}^{\ell \ell}$ and $C_{7}^{s b}$, as is depicted to the left in Fig. 1 . Due to the correlation with $b \rightarrow c \tau v$, these Wilson coefficients can be expressed as functions of $R\left(D^{(*)}\right) / R\left(D^{(*)}\right)_{\mathrm{SM}}$. The Wilson coefficients' dependency on these ratios is shown in the right plot of Fig. 1, where the RGE evolution of $C_{7}^{s b}$ from the NP scale down to the $b$ quark scale is also taken into account (see Ref. [40]). Interestingly, assuming an explanation of $b \rightarrow c \tau v$ data, the effects 


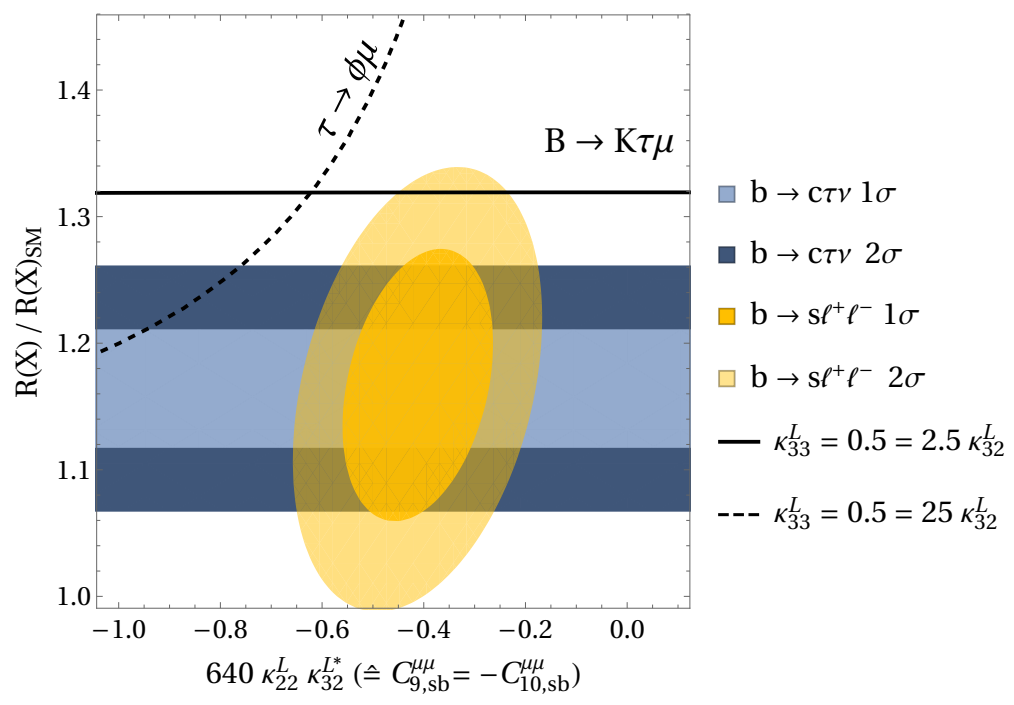

Figure 2: Allowed (colored) regions in the $C_{9, s b}^{\mu \mu}=-C_{10, s b}^{\mu \mu}\left(\equiv 640 \kappa_{22}^{L} \kappa_{32}^{L *}\right)-R(X) / R(X)_{\mathrm{SM}}$ plane for $M=1$ $\mathrm{TeV}$ and $X=\left\{D, D^{*}\right\}$ at the $1 \sigma$ and $2 \sigma$ level. The region above the black dashed (solid) line is excluded by $\tau \rightarrow \phi \mu(B \rightarrow K \tau \mu))$ for $\kappa_{33}^{L}=0.5=25 \kappa_{32}^{L}\left(\kappa_{33}^{L}=0.5=2.5 \kappa_{32}^{L}\right)$. The bound from $\tau \rightarrow \phi \mu(B \rightarrow$ $K \tau \mu$ ) depends on $\kappa_{33}^{L}$ and $\kappa_{32}^{L}$ and gets stronger if $\kappa_{32}^{L}$ gets smaller (larger). That is, for $\kappa_{33}^{L}=0.5$ and $2.7 \lesssim \kappa_{33}^{L} / \kappa_{32}^{L} \lesssim 27$, the whole $2 \sigma$ region preferred by $b \rightarrow c \tau \nu$ and $b \rightarrow s \ell^{+} \ell^{-}$data is consistent with these bounds. Note that we used the most recent experimental results for both the $b \rightarrow c \tau v$ and $b \rightarrow s \ell^{+} \ell^{-}$ transitions, therefore updating our analysis in Ref. [40].

generated in $C_{9, s b}^{\ell \ell}$ and $C_{7}^{s b}$ agree with the $1 \sigma$ ranges of the model independent fit to $b \rightarrow s \mu^{+} \mu^{-}$ data excluding LFU violating observables $[38,41]$.

Now we also allow $\kappa_{32}^{L}$ and $\kappa_{22}^{L}$ to be non-zero, generating a tree level effect in $b \rightarrow s \mu^{+} \mu^{-}$ which is necessary to account for the LFU violating observables as well. In Fig. 2 we show the allowed (colored) regions from $b \rightarrow s \mu^{+} \mu^{-}$and $b \rightarrow c \tau \nu$ as well as the exclusions from $b \rightarrow s \tau \mu$ and $\tau \rightarrow \phi \mu$. A simultaneous explanation of the anomalies is perfectly possible since the colored regions overlap and do not extend to the parameter space excluded by $b \rightarrow s \tau \mu$ and $\tau \rightarrow \phi \mu$. Interestingly, we predict a lepton flavor universal effect in $C_{9, s b}^{\ell \ell}$ and $C_{7}^{s b}$ in addition to a LFU violating tree-level effect of the form $C_{9, s b}^{\mu \mu}=-C_{10, s b}^{\mu \mu}$ in muonic channels only. This means that the effect of NP compared to the SM is expected to be larger in lepton flavor universal observables like $P 5^{\prime}$ relative to LFU violation observables as $R\left(K^{(*)}\right)$, which is in perfect agreement with global fit scenarios [8]. In fact, the agreement is even better after the inclusion of the new measurements of BELLE and LHCb.

\section{References}

[1] A. Crivellin, C. Greub, D. Müller and F. Saturnino, Phys. Rev. Lett. 122, no. 1, 011805 (2019) doi:10.1103/PhysRevLett.122.011805 [arXiv:1807.02068 [hep-ph]].

[2] R. Aaij et al. [LHCb Collaboration], Phys. Rev. Lett. 122, no. 19, 191801 (2019) doi:10.1103/PhysRevLett.122.191801 [arXiv:1903.09252 [hep-ex]].

[3] A. Abdesselam et al. [Belle Collaboration], arXiv:1904.02440 [hep-ex]. 
[4] A. Abdesselam et al. [Belle Collaboration], arXiv:1904.08794 [hep-ex].

[5] R. Aaij et al. [LHCb Collaboration], JHEP 1708, 055 (2017) doi:10.1007/JHEP08(2017)055 [arXiv:1705.05802 [hep-ex]].

[6] R. Aaij et al. [LHCb Collaboration], JHEP 1602, 104 (2016) doi:10.1007/JHEP02(2016)104 [arXiv:1512.04442 [hep-ex]].

[7] B. Capdevila, A. Crivellin, S. Descotes-Genon, J. Matias and J. Virto, JHEP 1801, 093 (2018) doi:10.1007/JHEP01(2018)093 [arXiv:1704.05340 [hep-ph]].

[8] M. Algueró, B. Capdevila, A. Crivellin, S. Descotes-Genon, P. Masjuan, J. Matias and J. Virto, arXiv:1903.09578 [hep-ph].

[9] J. Aebischer, W. Altmannshofer, D. Guadagnoli, M. Reboud, P. Stangl and D. M. Straub, arXiv:1903.10434 [hep-ph].

[10] M. Ciuchini, A. M. Coutinho, M. Fedele, E. Franco, A. Paul, L. Silvestrini and M. Valli, arXiv:1903.09632 [hep-ph].

[11] A. Arbey, T. Hurth, F. Mahmoudi, D. M. Santos and S. Neshatpour, arXiv:1904.08399 [hep-ph].

[12] R. Alonso, B. Grinstein and J. Martin Camalich, JHEP 1510, 184 (2015) doi:10.1007/JHEP10(2015)184 [arXiv:1505.05164 [hep-ph]].

[13] L. Calibbi, A. Crivellin and T. Ota, Phys. Rev. Lett. 115, 181801 (2015) doi:10.1103/PhysRevLett.115.181801 [arXiv:1506.02661 [hep-ph]].

[14] S. Fajfer and N. Konik, Phys. Lett. B 755, 270 (2016) doi:10.1016/j.physletb.2016.02.018 [arXiv:1511.06024 [hep-ph]].

[15] R. Barbieri, G. Isidori, A. Pattori and F. Senia, Eur. Phys. J. C 76, no. 2, 67 (2016) doi:10.1140/epjc/s10052-016-3905-3 [arXiv:1512.01560 [hep-ph]].

[16] R. Barbieri, C. W. Murphy and F. Senia, Eur. Phys. J. C 77, no. 1, 8 (2017) doi:10.1140/epjc/s10052-016-4578-7 [arXiv:1611.04930 [hep-ph]].

[17] G. Hiller, D. Loose and K. Schönwald, JHEP 1612, 027 (2016) doi:10.1007/JHEP12(2016)027 [arXiv:1609.08895 [hep-ph]].

[18] B. Bhattacharya, A. Datta, J. P. Guévin, D. London and R. Watanabe, JHEP 1701, 015 (2017) doi:10.1007/JHEP01(2017)015 [arXiv:1609.09078 [hep-ph]].

[19] D. Buttazzo, A. Greljo, G. Isidori and D. Marzocca, JHEP 1711, 044 (2017) doi:10.1007/JHEP11(2017)044 [arXiv:1706.07808 [hep-ph]].

[20] J. Kumar, D. London and R. Watanabe, Phys. Rev. D 99, no. 1, 015007 (2019) doi:10.1103/PhysRevD.99.015007 [arXiv:1806.07403 [hep-ph]].

[21] N. Assad, B. Fornal and B. Grinstein, Phys. Lett. B 777, 324 (2018) doi:10.1016/j.physletb.2017.12.042 [arXiv:1708.06350 [hep-ph]].

[22] L. Di Luzio, A. Greljo and M. Nardecchia, Phys. Rev. D 96, no. 11, 115011 (2017) doi:10.1103/PhysRevD.96.115011 [arXiv:1708.08450 [hep-ph]].

[23] L. Calibbi, A. Crivellin and T. Li, Phys. Rev. D 98, no. 11, 115002 (2018) doi:10.1103/PhysRevD.98.115002 [arXiv:1709.00692 [hep-ph]]. 
[24] M. Bordone, C. Cornella, J. Fuentes-Martin and G. Isidori, Phys. Lett. B 779, 317 (2018) doi:10.1016/j.physletb.2018.02.011 [arXiv:1712.01368 [hep-ph]].

[25] R. Barbieri and A. Tesi, Eur. Phys. J. C 78, no. 3, 193 (2018) doi:10.1140/epjc/s10052-018-5680-9 [arXiv:1712.06844 [hep-ph]].

[26] M. Blanke and A. Crivellin, Phys. Rev. Lett. 121, no. 1, 011801 (2018) doi:10.1103/PhysRevLett.121.011801 [arXiv:1801.07256 [hep-ph]].

[27] A. Greljo and B. A. Stefanek, Phys. Lett. B 782, 131 (2018) doi:10.1016/j.physletb.2018.05.033 [arXiv:1802.04274 [hep-ph]].

[28] M. Bordone, C. Cornella, J. Fuentes-Martín and G. Isidori, JHEP 1810, 148 (2018) doi:10.1007/JHEP10(2018)148 [arXiv:1805.09328 [hep-ph]].

[29] S. Matsuzaki, K. Nishiwaki and K. Yamamoto, JHEP 1811, 164 (2018) doi:10.1007/JHEP11(2018)164 [arXiv:1806.02312 [hep-ph]].

[30] L. Di Luzio, J. Fuentes-Martin, A. Greljo, M. Nardecchia and S. Renner, JHEP 1811, 081 (2018) doi:10.1007/JHEP11(2018)081 [arXiv:1808.00942 [hep-ph]].

[31] A. Biswas, D. Kumar Ghosh, N. Ghosh, A. Shaw and A. K. Swain, arXiv:1808.04169 [hep-ph].

[32] A. Angelescu, D. Beirevi, D. A. Faroughy and O. Sumensari, JHEP 1810, 183 (2018) doi:10.1007/JHEP10(2018)183 [arXiv:1808.08179 [hep-ph]].

[33] M. Algueró, B. Capdevila, S. Descotes-Genon, P. Masjuan and J. Matias, Phys. Rev. D 99, no. 7, 075017 (2019) doi:10.1103/PhysRevD.99.075017 [arXiv:1809.08447 [hep-ph]].

[34] Y. Amhis et al. [HFLAV Collaboration], Eur. Phys. J. C 77, no. 12, 895 (2017) doi:10.1140/epjc/s10052-017-5058-4 [arXiv:1612.07233 [hep-ex]].

[35] R. Aaij et al. [LHCb Collaboration], Phys. Rev. Lett. 120, no. 12, 121801 (2018) doi:10.1103/PhysRevLett.120.121801 [arXiv:1711.05623 [hep-ex]].

[36] J. C. Pati and A. Salam, Phys. Rev. D 10, 275 (1974) Erratum: [Phys. Rev. D 11, 703 (1975)]. doi:10.1103/PhysRevD.10.275, 10.1103/PhysRevD.11.703.2

[37] C. Cornella, J. Fuentes-Martin and G. Isidori, arXiv:1903.11517 [hep-ph].

[38] S. Descotes-Genon, L. Hofer, J. Matias and J. Virto, JHEP 1606, 092 (2016) doi:10.1007/JHEP06(2016)092 [arXiv:1510.04239 [hep-ph]].

[39] B. Capdevila, A. Crivellin, S. Descotes-Genon, L. Hofer and J. Matias, Phys. Rev. Lett. 120, no. 18, 181802 (2018) doi:10.1103/PhysRevLett.120.181802 [arXiv:1712.01919 [hep-ph]].

[40] A. Crivellin and F. Saturnino, arXiv:1905.08257 [hep-ph].

[41] W. Altmannshofer and D. M. Straub, Eur. Phys. J. C 75, no. 8, 382 (2015) doi:10.1140/epjc/s10052-015-3602-7 [arXiv:1411.3161 [hep-ph]]. 